\title{
Cornutus
}

\section{Die Griechischen Götter}

Ein Überblick über Namen, Bilder und Deutungen

Hrsg. v. Heinz-Günther Nesselrath, eingel., übers. u. m. interpretierenden Essays vers. v. Fabio Berdozzo, George Boys-Stones, Hans-Josef Klauck, Ilaria Ramelli u. Alexei V. Zadorojnyi

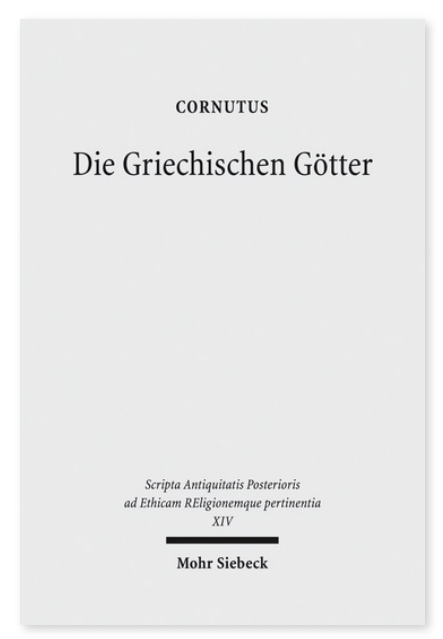

2009. X, 259 Seiten. SAPERE XIV

ISBN 978-3-16-156441-3

DOI 10.1628/978-3-16-156441-3

eBook PDF

ISBN 978-3-16-150071-8

Broschur 29,00€

ISBN 978-3-16-150072-5

Leinen $59,00 €$
Lucius Annaeus Cornutus stammte aus Leptis Magna in Libyen und lebte zur Zeit Kaiser Neros als stoischer Philosoph in Rom. Von seinen Werken (Schriften zur Rhetorik und Philosophie; Kommentar zum Dichter Vergil) ist vollständig nur die hier erstmals in deutscher Übersetzung vorgelegte Epidromê tôn kata tên Hellênikên theologian paradedomenôn ('Überblick über das in der griechischen Götterlehre Überlieferte') erhalten. Dieses Werk stellt ein ebenso einzig- wie eigenartiges Handbuch der stoischen allegorischen Götterdeutung dar, das für jede griechische Gottheit, von Uranos bis Hades, eine etymologische und allegorische Deutung ihrer Namen, Epitheta und Attribute sowie einiger Aspekte der mit ihnen verbundenen Mythen, Riten und bildlichen Darstellungen liefert. Damit bildet es einen bemerkenswerten Versuch, den klassischen antiken Polytheismus mit Hilfe einer entwickelten Philosophie zu deuten.

Unter Mitarbeit von:

Fabio Berdozzo, George Boys-Stones, Hans-Josef Klauck, Ilaria Ramelli und Alexey Zadorojnjy

Cornutus Keine aktuellen Daten verfügbar.

Fabio Berdozzo Geboren 1976; 2000 Magister (»diploma di laurea«) an der Universität Padua, Italien; 2007 Promotion in Klassischer Philologie an der Georg-August-Universität, Göttingen; seit 2009 Dozent für Griechisch und Latein (Nachfolge Dr. K.-H. Pridick) an der Kirchlichen Hochschule Wuppertal/Bethel.

George Boys-Stones is currently Professor of Classics and Philosophy at Toronto University.

Hans-Josef Klauck Geboren 1946; 1977 Promotion; 1980 Habilitation; 1982-97 Professor in Würzburg; 1997-2001 Professor in München, 1999-2003 Honorarprofessor an der Universität Pretoria/Südafrika; ab 2001 Full Professor, ab 2006 NaomiShenstone-Donnelley Professor an der Divinity School der University of Chicago; 2016 emeritiert.

Ilaria Ramelli Born 1973; earned two MAs, a PhD, a postdoc, and two Habilitations to Full Professor (History of Philosophy and Ancient Greek); Full Professor of Theology and K. Britt Chair (Graduate School of Theology, SHMS, Angelicum University), Senior Visiting Professor of Greek Thought, and the Director of International Research Projects.

Alexei Zadorojnyi is currently lecturer for Archaeology, Classics and Egyptology at Liverpool University.

Jetzt bestellen:

https://mohrsiebeck.com/buch/die-griechischen-goetter-9783161564413?no_cache=1

order@mohrsiebeck.com

Telefon: +49 (0)7071-923-17

Telefax: $+49(0) 7071-51104$ 